\title{
Defining Desirable Central Nervous System Drug Space through the Alignment of Molecular Properties, in Vitro ADME, and Safety Attributes
}

\author{
Travis T. Wager, ${ }^{* \dagger, *}$ Ramalakshmi Y. Chandrasekaran, ${ }^{*}$ Xinjun Hou, ${ }^{\dagger, *}$ \\ Matthew D. Troutman, ${ }^{\S}$ Patrick R. Verhoest, ${ }^{+,}$Anabella Villalobos, ${ }^{\dagger, *}$ and \\ Yvonne Will ${ }^{\perp}$ \\ NNeuroscience Medicinal Chemistry, ${ }^{\S}$ Department of Pharmacokinetics, Dynamics and Metabolism Screening CoE, and \\ ${ }^{\perp}$ Compound Safety Prediction, Pfizer PharmaTherapeutics Research and Development, 558 Eastern Point Road, Groton, Connecticut 06340
}

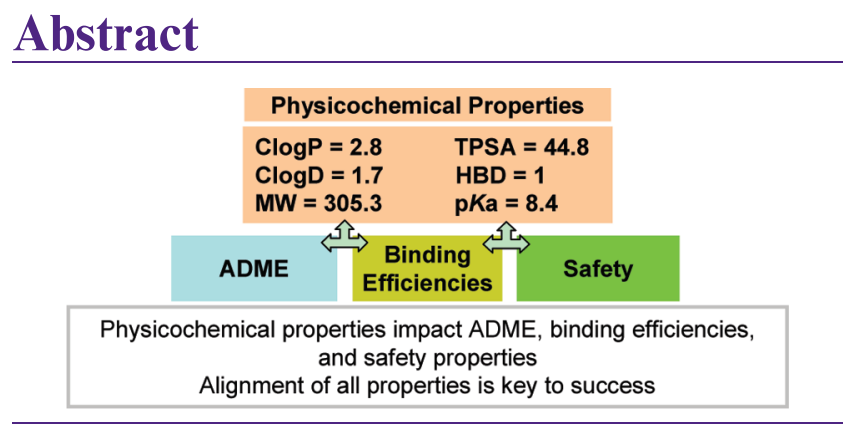

As part of our effort to increase survival of drug candidates and to move our medicinal chemistry design to higher probability space for success in the Neuroscience therapeutic area, we embarked on a detailed study of the property space for a collection of central nervous system (CNS) molecules. We carried out a thorough analysis of properties for 119 marketed CNS drugs and a set of 108 Pfizer CNS candidates. In particular, we focused on understanding the relationships between physicochemical properties, in vitro ADME (absorption, distribution, metabolism, and elimination) attributes, primary pharmacology binding efficiencies, and in vitro safety data for these two sets of compounds. This scholarship provides guidance for the design of CNS molecules in a property space with increased probability of success and may lead to the identification of druglike candidates with favorable safety profiles that can successfully test hypotheses in the clinic.

Keywords: Central nervous system (CNS), CNS drugs, CNS candidates, lipophilicity, topological polar surface area, polarity, molecular weight, hydrogen bond donor, most basic $\mathrm{p} K_{\mathrm{a}}$, high-throughput screening, passive permeability, Madin-Darby canine kidney, P-glycoprotein, human liver microsome stability, unbound intrinsic clearance, ligand efficiency, ligand-lipophilicity efficiency, ligand-efficiencydependent lipophilicity, drug-drug interactions, dofetilide binding, transformed human liver epithelial cells, cellular toxicity
$\mathrm{T}$ he pharmaceutical industry faces significant attrition of drug candidates due to safety or suboptimal pharmacokinetics findings, resulting in increased costs and extended timelines for the drug development process (1). This paradigm is unsustainable, and there is an urgent need to identify new strategies to decrease cycle times in discovery, to increase survival of drug candidates, and to expedite validation of new mechanistic hypotheses in the clinic, which may result in the launch of more new drugs. Ideally, medicinal chemists would build in druglike properties and desirable safety attributes at the design stage, before molecules are synthesized. As part of our effort to increase survival of drug candidates and to move our medicinal chemistry design to higher probability space for success in the Neuroscience therapeutic area, we embarked on data collection and rigorous analysis of the property space for central nervous system (CNS) molecules.

Numerous analyses of marketed drugs, clinical candidates, patented molecules, and CNS permeability have been reported in the literature (2-10). Our scholarship focused on a rigorous data generation and a thorough analysis of attributes for marketed CNS drugs (drug set or drugs) and a set of Pfizer CNS candidates (candidate set or candidates). In particular, we focused on understanding the interrelationships between physicochemical properties, in vitro ADME (absorption, distribution, metabolism, and elimination) attributes, primary pharmacology binding efficiencies, and in vitro safety data for these two sets of compounds. This data was examined with the goal of identifying trends and defining a set of property values that would best define the CNS drug space associated with a higher probability of clinical success. The knowledge obtained from this analysis could in turn be utilized prospectively to design safe and brain-penetrable molecules based on the alignment of a set of key properties.

Received Date: January 29, 2010

Accepted Date: March 2, 2010

Published on Web Date: March 25, 2010 
Physicochemical properties associated with druglike molecules have been described previously $(7,11,12)$. The well-known rule of five (RO5) was derived in 1997 from a database of clinical candidates that had reached phase II trials or further (13). It defined end points for a set of four physicochemical properties that described $90 \%$ of orally active drugs that achieved phase II clinical status: (a) molecular weight, MW $<500 \mathrm{Da}$; (b) lipophilicity, $\log P$ or the calculate of 1-octanol-water partition coefficient, $\mathrm{Clog} \mathrm{P}<5$; (c) number of hydrogen-bond donors, $\mathrm{OH}$ plus $\mathrm{NH}$ count, $<5$; and (d) number of hydrogen-bond acceptors, $\mathrm{O}$ plus $\mathrm{N}$ atoms, $<10$. These four physicochemical parameters and their criteria describe fundamental attributes that are associated with acceptable aqueous solubility and intestinal permeability, key factors for the first step of oral bioavailability (13). The RO5 was created to help medicinal chemists design compounds with improved physicochemical properties; its simplicity and predictability have resulted in its use as a standard benchmark of druglikeness. To go beyond the properties associated with the RO5, we became interested in developing a holistic understanding of physicochemical property space for CNS molecules by carrying out a thorough analysis of properties for CNS drugs and a set of CNS candidates.

In vitro $\mathrm{ADME}$ end points have become a main staple of information to guide medicinal chemists in drug design $(7,14,15)$. In an effort to gain a better perspective on ADME space for the drugs and the candidates, these molecules were profiled utilizing high-throughput (HT) assays commonly employed by Pfizer and the industry. The ADME assay end points that we focused on were passive apparent permeability $\left(P_{\text {app }}\right)$, P-glycoprotein (P-gp) efflux liability, and human liver microsomal (HLM) metabolic stability. We were interested in defining and understanding these ADME parameters with the idea that applying this knowledge prospectively at the drug design stage would yield compounds with reduced P-gp liability and low human dose projections.

Over the past few years, the relationships between in vitro potency, size, and lipophilicity have been examined and reviewed $(6,16)$. It has been realized that design by simply driving to a higher potency space is often futile, because it tends to result in merely increasing size or lipophilicity. Concepts such as ligand efficiency (LE), which correlates potency and size, and ligand-lipophilicity efficiency (LLE), which correlates potency and lipophilicity, have been utilized by medicinal chemists to retrospectively evaluate compound potency to properties. More recently, Keserü and Makara proposed the use of ligand-efficiency-dependent lipophilicity (LELP), a function that correlates all three factors of potency, size, and lipophilicity (17). Defining in vitro potency space in relationship to the physicochemical properties of successful drugs and candidates may expand our understanding of the optimum LE, LLE, and LELP values in CNS space. Furthermore, connecting in vitro potency to physicochemical properties such as $\mathrm{Clog} \mathrm{P}$ and size (MW) will enable better utilization of these easily calculated properties in CNS drug design.

Safety findings are one of the most prevalent factors in compound attrition (1). As part of our analysis of properties for drugs and candidates, we became interested in understanding profiles associated with certain safety parameters. Utilizing HT assays, we generated in vitro data to assess potential for the following safety risks: drug-drug interactions (CYP inhibitions), hERG liability (inhibition of dofetilide binding), and cellular toxicity. In addition, we hoped to develop an improved understanding of key physicochemical properties associated with toxicity outcomes. Hughes et al., in examining physicochemical drug properties associated with in vivo toxicology outcomes, have confirmed a long-standing empirical observation of increased incidence of toxicity for highly lipophilic and low polarity compounds (18): an increased relative risk (6:1) for an adverse event in toxicology studies was observed when compounds possessed both high lipophilicity $(\mathrm{Clog} \mathrm{P}>3)$ and low topological polar surface area (TPSA $<75 \AA^{2}$ ). The outcome from their analysis supports the claim that the increase in in vivo toxicity may be driven by the promiscuity of highly lipophilic molecules (6). The ClogP $>3$ and TPSA $<75$ relative risk factors ( 3 and 75 RRFs) can inform medicinal chemists about safety and the probability of succeeding in a particular chemical space. On the other hand, a crucial consideration in the design of CNS active compounds is brain permeability, and a common approach has been to improve brain penetration by driving compounds toward this exact higher-risk area of increased lipophilicity and lower polarity. Based on the 3 and 75 RRFs, this approach could place a substantial proportion of investigational CNS compounds in a chemical space with increased risk of in vivo toxicology findings. By understanding key safety and pharmacokinetic parameters, we hoped to identify strategies to balance these characteristics, and to drive the molecular design to the space of higher probability of success from both the brain penetration and safety perspectives.

In this paper, we report our analysis of physicochemical properties, in vitro ADME attributes, binding efficiencies, and in vitro safety assay data associated with 119 drugs and 108 candidates and its utility in identifying the most favorable CNS property space. We generated a significant amount of data in-house to carry out this analysis and built the scholarship around design strategies that may lead to successful CNS drug candidates. 

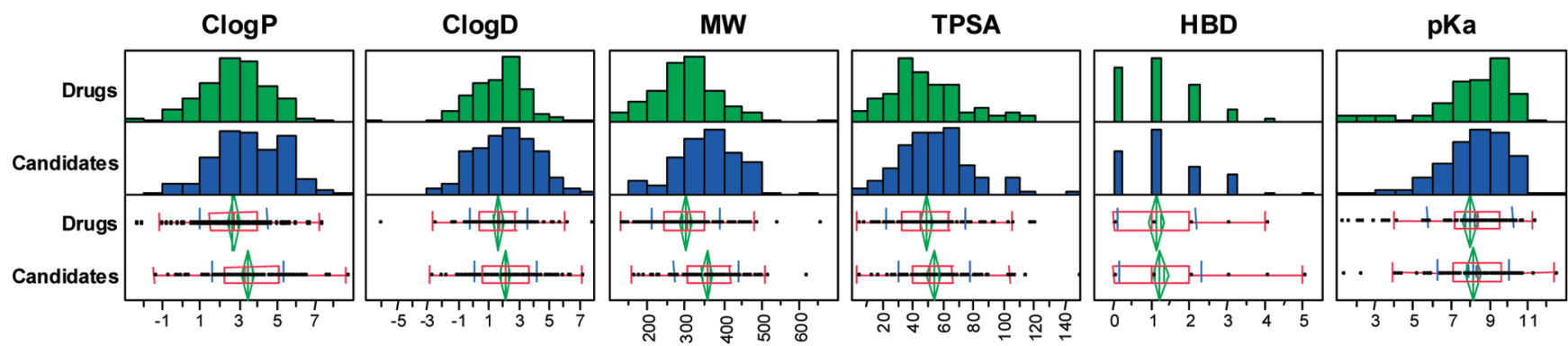

\begin{tabular}{|c|c|c||c|c|c|}
\hline & Drugs & Candidates \\
\hline $\mathbf{1 0 \%}$ & 0.4 & 1.2 & $\mathbf{1 0 \%}$ & -0.5 & -0.6 \\
\hline $\mathbf{2 5 \%}$ & 1.5 & 2.3 \\
\hline Median & 2.8 & 3.3 \\
\hline $\mathbf{7 5 \%}$ & 4.0 & 5.1 & 0.4 & 0.7 \\
\hline $\mathbf{9 0 \%}$ & 5.1 & 5.8 & $\mathbf{M e d i a n}$ & 1.7 & 2.2 \\
\hline $\mathbf{N}$ & 119 & 108 & $\mathbf{7 0 \%}$ & 2.8 & 3.7 \\
\hline Mean & 2.7 & 3.5 \\
\hline S.E.M. & 0.2 & 0.2 & $\mathbf{N}$ & 119 & 108 \\
\hline \multicolumn{2}{|c|}{ Prob $>|\mathbf{t}|$} & 0.0022 \\
\hline
\end{tabular}

\begin{tabular}{|c|c|c|}
\hline & Drugs & Candidates \\
\hline $\mathbf{1 0} \%$ & 181.2 & 252.5 \\
\hline $\mathbf{2 5 \%}$ & 246.4 & 305.7 \\
\hline Median & 305.3 & 360.4 \\
\hline $\mathbf{7 5 \%}$ & 350.5 & 419.6 \\
\hline $\mathbf{9 0 \%}$ & 426.5 & 463.0 \\
\hline $\mathbf{N}$ & 119 & 108 \\
\hline Mean & 303.5 & 357.4 \\
\hline S.E.M. & 8.2 & 8.1 \\
\hline Prob > |t| & $<0.0001$ \\
\hline
\end{tabular}

\begin{tabular}{|c|c|c|}
\hline & Drugs & Candidates \\
\hline $\mathbf{1 0 \%}$ & 16.1 & 21.3 \\
\hline $\mathbf{2 5 \%}$ & 32.3 & 39.5 \\
\hline Median & 44.8 & 51.2 \\
\hline $\mathbf{7 5 \%}$ & 63.3 & 66.1 \\
\hline $\mathbf{9 0 \%}$ & 86.2 & 80.9 \\
\hline $\mathbf{N}$ & 119 & 108 \\
\hline Mean & 48.5 & 53.6 \\
\hline S.E.M. & 2.3 & 2.3 \\
\hline Prob > |t| & 0.1171 \\
\hline
\end{tabular}

\begin{tabular}{|c|c|c||c|c|c|}
\hline & Drugs & Candidates & & Drugs & Candidates \\
\hline $\mathbf{1 0 \%}$ & 0 & 0 & $\mathbf{1 0 \%}$ & 3.9 & 5.7 \\
\hline $\mathbf{2 5 \%}$ & 0 & 0 & $\mathbf{2 5 \%}$ & 7.2 & 7.1 \\
\hline Median & 1 & 1 & Median & 8.4 & 8.4 \\
\hline $\mathbf{7 5 \%}$ & 2 & 2 & $\mathbf{7 5 \%}$ & 9.5 & 9.7 \\
\hline $\mathbf{9 0 \%}$ & 3 & 3 & $\mathbf{9 0 \%}$ & 10.1 & 10.3 \\
\hline $\mathbf{N}$ & 119 & 108 & $\mathbf{N}$ & 96 & 99 \\
\hline Mean & 1.1 & 1.2 & Mean & 8.0 & 8.1 \\
\hline S.E.M. & 0.09 & 0.1 & S.E.M. & 0.2 & 0.2 \\
\hline Prob $>$ |t| & 0.4877 & Prob > |t| & 0.6272 \\
\hline
\end{tabular}

Figure 1. Physicochemical property distribution and statistics of drugs and candidates are shown for ClogP, ClogD, MW, TPSA, HBD, and $\mathrm{p} K_{\mathrm{a}} . N$ represents the number of compounds included in each analysis. Two-sided Student's $t$ test was applied to evaluate statistical significances of drugs and candidates.

\section{Results and Discussion}

\section{Physicochemical Property Space for CNS Mole- cules}

The 119 drugs and 108 candidates were evaluated against a set of six calculated fundamental physicochemical properties that have gained wide acceptance in the medicinal chemistry community as key parameters for drug design: (a) lipophilicity, calculated partition coefficient $(\mathrm{C} \log \mathrm{P})$; (b) distribution coefficient at $\mathrm{pH}=7.4(\mathrm{ClogD})$; (c) molecular weight (MW); (d) topological polar surface area (TPSA) (19); (e) number of hydrogen bond donors (HBD); (f) most basic center $\left(\mathrm{p} K_{\mathrm{a}}\right)(4,13,19,20)$. The range of physicochemical properties of the drugs and candidates as described by these six parameters was quite broad (Figure 1). The $\mathrm{C} \log \mathrm{P}$ values for the majority of the drugs varied from 0.4 (10th percentile) to 5.1 (90th percentile) with a median ClogP value of 2.8. As expected for CNS drugs, a similar but shifted range existed for $\mathrm{ClogD}$, which varied from -0.5 (10th percentile) to 3.8 (90th percentile) with a median value of 1.7. Compounds in the candidate set showed similar a $\mathrm{Clog} P$ range, but right-shifted to higher lipophilicity, with 10th to 90th percentile values of 1.2 to 5.8 and a higher median value of 3.3, which was statistically significant (two-sided Student's $t$ test, $p=0.0022$ ). There was no statistically significant difference $(p=0.057)$ in ClogD between the two sets, although the drug set had a lower median value by 0.5 units. Comparison of the MW for drugs and candidates also showed that the candidate set had a higher median MW (360.4) than the drug set (305.3) by $55 \mathrm{Da}$, which was statistically significant $(p<0.0001)$.
Our analysis suggests that for centrally acting drugs, there may be a need to design compounds with further reduced $\mathrm{ClogP}$ or MW to better match the corresponding properties in the drug set.

Polarity, as described by polar surface area (TPSA), ranged from about $16.1 \AA^{2}$ (10th percentile) to $86.2 \AA^{2}$ (90th percentile) with a median value of $44.8 \AA^{2}$ for the drug set. There was no significant difference between candidates and drugs. TPSA values for both sets of compounds were higher than anticipated with approximately $25 \%$ of the drugs having TPSA $>63 \AA^{2}$ (75th percentile) and more than $10 \%$ of the drugs having TPSA > $86 \AA^{2}$. The drugs and the candidates had a minimal number of hydrogen bond donors (HBD), with the median value being one (1) hydrogen bond donor and with $85 \%$ of the compounds in both sets having HBD $\leq 2$. Lipinski's RO5 identified HBD as a critical component of the drug property analysis and targets a HBD count ( $\mathrm{OH}$ plus NH count) of $<5$. Based on the number of HBD associated with CNS drugs and candidates, optimization of HBD to $\leq 2$ may increase the odds of identifying CNS-penetrable compounds.

Many CNS compounds have basic amines as part of their pharmacophore, and it is known that there are safety liabilities associated with lipophilic basic amines, such as hERG inhibition (7) and phospholipidosis (21); this prompted our interest in understanding basicity profiles. The $\mathrm{p} K_{\mathrm{a}}$ profiles of the drugs and candidates were similar, with a median $\mathrm{p} K_{\mathrm{a}}$ of 8.4 for both the drug and the candidate sets. It is interesting to note that only about $10 \%$ of the drugs have a $\mathrm{p} K_{\mathrm{a}}>10$ and that there are numerous (25/119) CNS drugs with $\mathrm{p} K_{\mathrm{a}} \leq 7.4$. 
While the CNS drug space as defined by ClogP, ClogD, MW, TPSA, HBD, and $\mathrm{p} K_{\mathrm{a}}$ is broad, our analysis points to optimal ranges for these properties (median values were found to be $\mathrm{Clog} \mathrm{P}=2.8, \mathrm{ClogD}=$ $1.7, \mathrm{MW}=305.3 \mathrm{Da}$, TPSA $=44.8 \AA^{2}, \mathrm{HBD}=1$, and $\mathrm{p} K_{\mathrm{a}}=8.4$ ), which may be of great utility in prospective design of CNS active molecules and the identification of drug candidates that can successfully progress to the clinic.

\section{ADME Profiles of CNS Molecules}

Significant advances in the development of HT in vitro ADME assays have enabled the earlier assessment of potential liabilities (low permeability, susceptibility to CNS efflux transporters, and metabolic instability) associated with new compounds; $10-20$ years ago, these assays did not exist or were not routinely used at a stage that informed drug design. In an effort to gain a better perspective on the ADME properties of drugs and candidates, we embarked on the in vitro profiling of these compounds in in-house HT assays to assess permeability, P-gp efflux liability, and metabolic stability. We have used the MDCK cell line to profile passive apparent permeability $\left(P_{\text {app }}\right)(14)$. The MDCK cell line is an epithelial cell line that spontaneously forms a confluent polarized monolayer, well-suited for determination of compound transport across epithelial and barrier tissues. From MDCK assay data, we classified the permeability of a molecule as low, moderate, or high based on its relative ranges of $P_{\text {app }}$ rates as follows: $P_{\text {app }} \leq 2.5$, low permeability $2.5<P_{\text {app }} \leq 10$, moderate permeability; and $P_{\text {app }}>10$, high permeability; units are $10^{-6} \mathrm{~cm} / \mathrm{s}$. The P-gp efflux liability was assessed utilizing the MDR1-MDCK cell line, an MDCK line stably transfected with the MDR1 gene, which expresses a functionally active human P-gp (14). Transport is determined in both directions, from apical or luminal to basolateral (A to B) and basolateral to apical or luminal (B to A) directions. Resultant $P_{\text {app }}$ values were then compared using eq 1 . On the basis of our historical data and accounting for experimental and cell line variance, a compound with an efflux ratio $(E R)>2.5$ is considered to be a P-gp substrate and may experience P-gp efflux liability (14).

$$
\text { P-gp ER }=\frac{P_{\text {app }}(\text { B to A })}{P_{\text {app }}(\text { A to } \mathrm{B})}
$$

We were able to obtain $P_{\text {app }}$ and P-gp efflux data for approximately $60-75 \%$ of compounds in the drug and candidate sets (Figure 2A,B), see Supporting Information for tabulated values. In the MDCK assay, $75 \%$ of the drugs show high $P_{\text {app }}$ values, while the candidates had a lower percentage $(51 \%)$ of compounds with high $P_{\text {app }}$ values $(p=0.0008)$. A similar discrepancy was observed when we assessed P-gp efflux liabilities for
A)

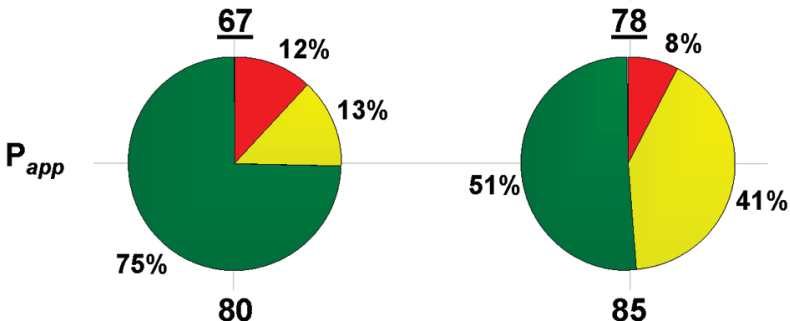

B)

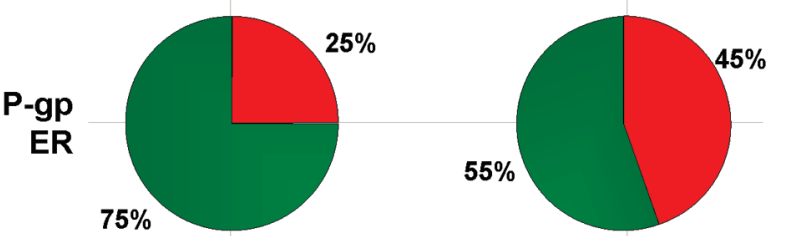

C)
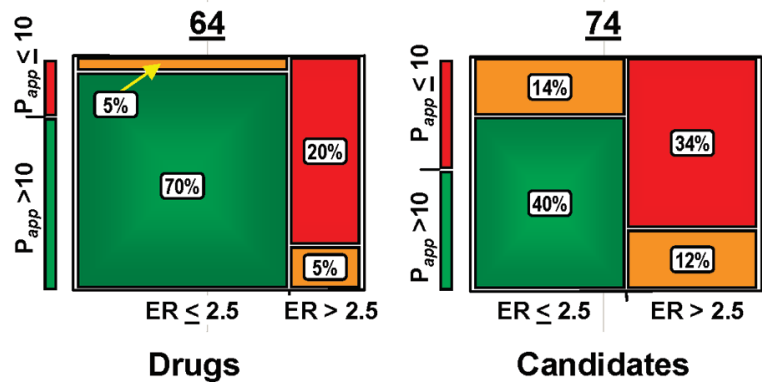

Figure 2. The distributions of $P_{\text {app }}$ and P-gp efflux ratio of drugs and candidates. The numbers of compounds with measured data are highlighted above each graph. (A) The binned values of $P_{\text {app }}$ obtained from the MDCK assay, color-coded by high permeability $\left(P_{\text {app }}>10\right.$, green), moderate permeability $\left(2.5<P_{\text {app }} \leq 10\right.$, yellow $)$, and low permeability $\left(P_{\text {app }} \leq 2.5\right.$, red $)$ in the units of $10^{-6} \mathrm{~cm} / \mathrm{s}$. (B) The binned values for P-gp efflux liability, color-coded by low P-gp liability (P-gp ER $\leq 2.5$, green) or high P-gp liability (P-gp ER $>2.5$, red). (C) Mosaic plots of molecules with both $P_{\text {app }}$ and P-gp data. Green blocks represent the percentages of molecules with both attributes (high $P_{\text {app }}$ and low P-gp liability), the orange blocks represent percentages of molecules with only one attribute (high $P_{\text {app }}$ or low P-pg liability), and the red blocks represent the molecules with neither high $P_{\text {app }}$ nor low P-gp.

both sets of compounds (Figure 2B). Profiling with the MDR1-MDCK assay showed that $75 \%$ of the drugs and $55 \%$ of the candidates were not considered to be Pgp efflux substrates, because their efflux ratios were $\leq$ 2.5 (Figure 2B). Consequently, there is a potentially lower possibility of brain penetration issues due to P-gp efflux liability for these compounds. An optimal molecule could be achieved if it is aligned in both end points, possessing both high $P_{\text {app }}$ and low P-gp efflux liability. Analyzing the compound set using both $P_{\text {app }}$ and P-gp data (Figure 2C) revealed that 70\% (45 out of 64) of the drugs have both attributes aligned, while $40 \%$ of candidates ( 30 out of 74 ) have both high permeability and low P-gp liability. Several drugs had $P_{\text {app }}$ values $>35 \times 10^{-6}$ $\mathrm{cm} / \mathrm{s}$, and no predicted P-gp efflux liability (efflux ratios $\leq 2.5$ ) as exemplified by midazolam, alprazolam, minaprine, and zolpidem. Overall, the drugs had higher $P_{\text {app }}$ values and lower P-gp efflux liability than the candidate 
set $(p=0.0077)$. Given today's availability of HT assays such as MDCK and MDR1-MDCK and the knowledge that the drug set has a higher percentage of compounds with both high $P_{\text {app }}$ and low P-gp liability, medicinal chemists should utilize these end points to drive design and selection of centrally acting compounds.

Metabolic stability data for the drugs and the candidates, expressed as unbound intrinsic clearance $\left(\mathrm{CL}_{\mathrm{int}, \mathrm{u}}\right)$, was generated using a HT in vitro human liver microsome (HLM) assay and an in silico model of estimated microsomal free fraction $\left(\mathrm{cF}_{\mathrm{u}, \mathrm{mic}}\right)$ according to eq 2 (22). The use of $\mathrm{CL}_{\text {int,u }}$ has been found to give a more accurate prediction of in vivo clearance than does $\mathrm{CL}_{\text {int,app }}$ and as such we have used this value for these analyses (23). A higher $\mathrm{CL}_{\text {int,u }}$ value suggests a faster rate of metabolism of free drug. $\mathrm{CL}_{\text {int,u }}$ data calculated using eq 2 were binned as follows: $\mathrm{CL}_{\text {int,u }} \leq 100 \mathrm{~mL} /(\mathrm{min} \cdot \mathrm{kg}$ ) (low clearance) and $\mathrm{CL}_{\text {int, u }}>100 \mathrm{~mL} /(\mathrm{min} \cdot \mathrm{kg})$ (high clearance). The cutoff of $\mathrm{CL}_{\text {int, u }}$ used in this analysis was selected to link metabolic stability as measured by the HLM assay to projected dose (eq 3). Assuming that drug metabolism is primarily driven by cytochrome P450s (CYPs), the $\mathrm{CL}_{\text {int,u }}$ can be used as a key component of dose projection using eq 3 (23). For a molecule with an efficacious static state unbound concentration $\left(C_{\mathrm{ss}, \mathrm{u}}\right)$ of $30 \mathrm{nM}, \mathrm{MW}=305$ (median of drug set, Figure 1$), 100 \%$ absorption $\left(f_{\mathrm{a}}=1\right)$, and targeting a dose $\leq 100 \mathrm{mg}$ for a $70 \mathrm{~kg}$ human in a dosing interval of $24 \mathrm{~h}$, the clearance $\mathrm{CL}_{\mathrm{int}, \mathrm{u}}$ would need to be $\leq 100 \mathrm{~mL} /\left(\mathrm{min} \cdot \mathrm{kg}\right.$ ) (for example, $\mathrm{CL}_{\text {int }}=50 \mathrm{~mL} /$ $(\mathrm{min} \cdot \mathrm{kg})$ and $\mathrm{cF}_{\mathrm{u} \text {,mic }}=0.5$ ).

$$
\mathrm{CL}_{\mathrm{int}, u}=\frac{\mathrm{CL}_{\mathrm{int}}}{\mathrm{CF}_{\mathrm{u}, \mathrm{mic}}}
$$

$\operatorname{dose}[\mathrm{mg}]=\frac{C_{\mathrm{ss}, \mathrm{u}}[\mathrm{nM}] \mathrm{CL}_{\text {int }}, \mathrm{u}[\mathrm{mL} /(\mathrm{min} \cdot \mathrm{kg})] \tau[\mathrm{min}]}{f_{\mathrm{a}}} \mathrm{MW}[\mathrm{Da}]$

$$
\times 70 \times 10^{-9}
$$

We were able to carry out HLM assays and then calculate $\mathrm{CL}_{\text {int,u }}$ values for 90 of 119 drugs and 93 of 108 candidates (see Supporting Information for tabulated results). Of the 90 drugs with experimental data, $71 \%$ were classified as having low clearance, with $\mathrm{CL}_{\text {int,u }} \leq$ $100 \mathrm{~mL} /(\mathrm{min} \cdot \mathrm{kg}$ ) (Figure 3). The candidate set in general had fewer compounds $(48 \%)$ in the low clearance group, and the distribution was statistically different from the drug set $(p=0.0016)$. Based on a strong correlation between $\mathrm{CL}_{\text {int,u }}$ and $\mathrm{ClogP}$, it is not surprising that the candidate set had, on average, higher $C_{\text {int,u }}$ values, which could have originated from the higher intrinsic clearance and lower microsomal free fraction (24). In most cases, low clearance should be desirable, because it would contribute to low dose projections, which in turn may result in compounds with better

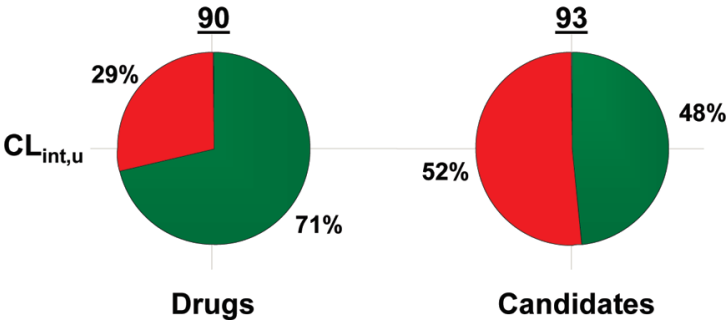

Figure 3. The distribution of binned clearance $\left(\mathrm{CL}_{\mathrm{int}, \mathrm{u}}\right)$ of drugs and candidates, color-coded by low clearance $\left(\mathrm{CL}_{\mathrm{int}, \mathrm{u}} \leq 100 \mathrm{~mL} /\right.$ $(\mathrm{min} \cdot \mathrm{kg})$, green) and high clearance $\left(\mathrm{CL}_{\mathrm{int}, \mathrm{u}}>100 \mathrm{~mL} /(\mathrm{min} \cdot \mathrm{kg})\right.$, red).

safety profiles. However, it is important to note that approximately one-third (29\%) of the drugs have high $\mathrm{CL}_{\text {int,u }}$ yet also have acceptable drug profiles because of compensation from other attributes. For example, paroxetine has a high $\mathrm{CL}_{\text {int,u }}(>100 \mathrm{~mL} /(\mathrm{min} \cdot \mathrm{kg}))$, but the clearance liability is overcome by its excellent potency $\left(K_{\mathrm{i}}=0.04 \mathrm{nM}\right)$ and efficient absorption from the gastrointestinal tract, resulting in a low dose (10$40 \mathrm{mg}$ ) compound (25). The paroxetine example is a good reminder that using a single end point such as $\mathrm{CL}_{\text {int,u }}$ to select a compound is unwise and can result in bypassing acceptable candidates.

\section{Alignment of ADME Attributes}

Examination of individual ADME properties $\left(P_{\text {app }}\right.$, P-gp, $C L_{\text {int,u }}$ ) suggested that for each property the drugs had on average a superior profile over the candidates. To increase the probability of success, design should focus on optimizing all properties in one molecule. To examine the alignment of these attributes, the number of attributes in the desirable space that each molecule possessed was summed using these criteria: high permeability $\left(P_{\text {app }}>10 \times 10^{-6} \mathrm{~cm} / \mathrm{s}\right)$, low P-gp liability $(\leq 2.5)$, and low clearance $\left(\mathrm{CL}_{\text {int, }}<100 \mathrm{~mL} /(\mathrm{min} \cdot \mathrm{kg})\right)$. A value of 3 was assigned to the molecule if all three criteria were satisfied, 2 was assigned if only two out of three attributes were satisfied, and so on. Using this simple scoring system, the drug set was highly populated $(61 \%)$ with compounds possessing all three desired ADME attributes (Figure 4). In contrast, the candidate set had statistically fewer compounds $(30 \%)$ with full ADME alignment $(p=0.0025)$. This analysis clearly suggests that a holistic alignment of attributes is key to increasing the probability of success; thus medicinal chemists should utilize all these attributes in designing new molecules.

\section{CNS Drugs and Candidates Have High LE, LLE, and LELP}

Concepts that correlate potency, size, and lipophilicity have been utilized by medicinal chemists to evaluate binding efficiencies to the desired biological target, with the goal of maximizing these efficiencies as new drug 
candidates are designed. Ligand efficiency (LE) is a concept used to estimate the efficiency of binding with respect to size, as measured by the number of heavy atoms (eq 4) (16). Ligand-lipophilicity efficiency (LLE) (eq 5) has also been proposed, suggesting that focus should be placed on maximizing lipophilicity efficiency (26). More recently, Keserü and Makara created a ligand-efficiency-dependent lipophilicity (LELP) descriptor, which factors in both size and lipophilicity

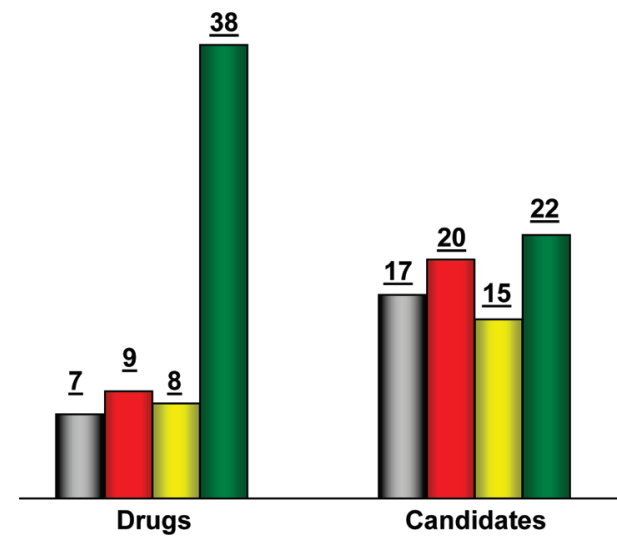

Figure 4. ADME attribute alignment of drugs and candidates, color-coded by the count of achieving the following criteria: high permeability $\left(P_{\text {app }}>10 \times 10^{-6} \mathrm{~cm} / \mathrm{s}\right)$, low P-gp liability $(\leq 2.5)$, and low clearance $\left(\mathrm{CL}_{\text {int, }} \leq 100 \mathrm{~mL} /(\mathrm{min} \cdot \mathrm{kg})\right)$. Colors are as follows: full alignment with $3 / 3$ attributes achieved (green), $2 / 3$ attributes achieved (yellow), 1/3 attributes achieved (red), 0/3 attributes achieved (gray). (eq 6) (17). Of the 119 drugs, we were able to obtain primary pharmacology potency values $\left(\mathrm{IC}_{50}\right.$ or $K_{\mathrm{i}}$ values) for 95 drugs based on literature reports. The median LE, LLE, and LELP values for the drug set were $0.52,6.3$, and 5.9, respectively (Figure 5). Median values obtained for the candidates were $0.46,6.4$, and 7.00 for LE, LLE, and LELP, respectively. There were no statistically significant differences between the LE and LLE values for the drugs and the candidates. Given the higher MW and $\mathrm{ClogP}$ values associated with the candidate set, on average the compounds in this set must be more potent than those in the drug set to generate equivalent LE and LLE median values. While the higher $\mathrm{MW}$ and $\mathrm{ClogP}$ values may have resulted in increased binding affinities and favorable LE and LLE values, these properties may have also contributed to the less favorable ADME profiles associated with the candidate set, as discussed previously. These observations reinforce the need to balance all properties in the prospective design of new molecules. The effects of higher lipophilicity and molecular weight are reflected in the LELP, see distribution LELP plot, Figure 5. Unlike the LE and LLE analyses, comparison of the median LELP values of drugs (5.9) to the candidates (7.0) yielded a statistically significant difference ( $p=$ 0.0066), suggesting that LELP maybe useful in postsynthesis analysis to ensure maximum binding efficiency for size and lipophilicity. Examination of the status (active or terminated) of the candidates with poor

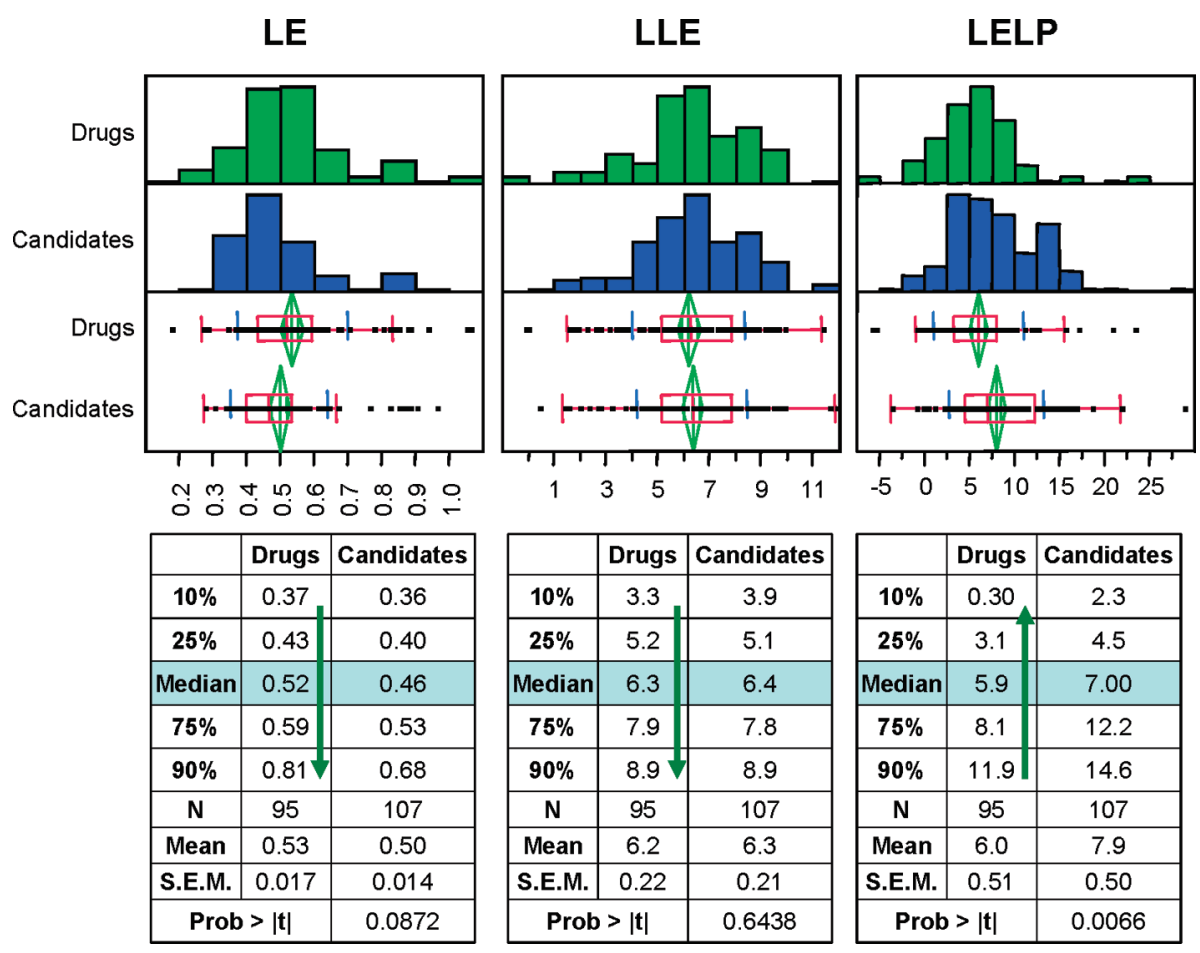

Figure 5. LE, LLE, and LELP distributions and statistics are shown for 95 drugs and 107 candidates with primary pharmacologic potency data. Two-sided Student's $t$ test was applied to test the difference between drugs and candidates. 


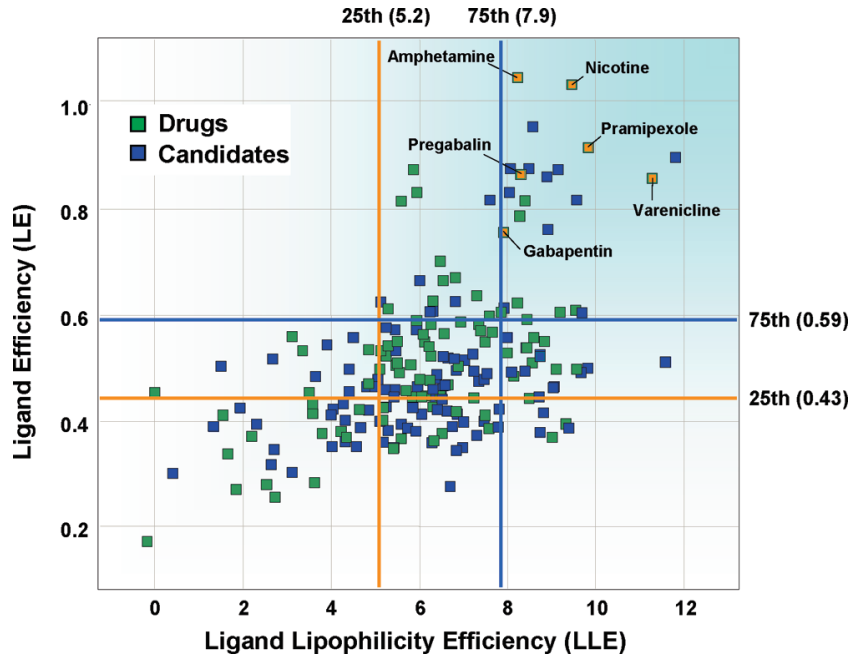

Figure 6. The relationship between ligand efficiency (LE) and ligandlipophilicity efficiency (LLE) of drugs and candidates is shown. The plot is guided by 25 th percentiles (orange lines) and 75 th percentiles (blue lines) of LE and LLE for the drug set. Compounds in the upper right square are compounds considered to have both high LE and LLE, and compounds occupying the lower left square are considered to have low LE and LLE.

LELP (LELP > 10) yielded only compounds that had been terminated along the drug discovery/development pathway.

$$
\begin{gathered}
\mathrm{LE}=\frac{-1.4 \log \left(K_{\mathrm{i}}[\mathrm{M}]\right)}{\text { number of heavy atoms }} \\
\mathrm{LLE}=-\log \left(K_{\mathrm{i}}[\mathrm{M}]\right)-\mathrm{Clog} \mathrm{D} \\
\mathrm{LELP}=\frac{\mathrm{Clog} \mathrm{P}}{\mathrm{LE}}
\end{gathered}
$$

The relationship between LE and LLE of the drugs and candidates is shown in Figure 6. Compounds with both high LE ( $\geq 75$ th percentile, $\geq 0.59$ ) and LLE $(\geq 75$ th percentile, $\geq 7.9$ ) values are captured in the upper right square and those with low efficiencies in the lower left square. Several drugs $(12 \%, 11 / 95)$ possess both high LE and LLE values as shown in Figure 6, upper right square. For example, recently launched varenicline (Chantix/Champix, $\alpha 4 \beta 2$ partial agonist) had LE and LLE values of 0.87 and 11.3 , respectively, a primary factor in its low dose value (1 $\mathrm{mg}$, once a day). Other drugs with high LE and LLE values were amphetamine (Adderall, NRI), pregabalin (Lyrica, $\alpha 2 \delta$ ), pramipexole (Mirapex, D2 agonist), nicotine (Nicorette, $\alpha 4 \beta 2$ ), and gabapentin (Neurontin, $\alpha 2 \delta$ ). This analysis reveals that a majority of the drugs $(75 \%)$ have either $L E$ value $\geq 0.43$ or LLE value $\geq 5.2$ and more than half $(54 \%)$ possess both. Thus, factoring binding efficiency factors such LE (size), LLE (lipophilicity), and LELP (size and lipophilicity) into the compound selection process will be a critical component for success.

\section{Safety End Points for Drugs and Candidates}

Early safety in vitro screening has become common practice within both large pharmaceutical and biotechnology companies (27). Examples of commonly measured end points to assess potential safety issues include inhibition of cytochrome P450 (CYPs) monooxygenase enzymes to determine potential for drug-drug interactions (28), inhibition of dofetilide binding (Dof) as a surrogate indicator of hERG potassium ion channel effects $(29,30)$, "off target" pharmacology utilizing broad ligand profiling assays (31), reactive metabolite generation, and genetic toxicity. In addition, in vitro cellular toxicity assays have been used as a surrogate for acute in vivo toxicity studies (32). As part of our analysis of properties for drugs and candidates, we generated in vitro data for these compounds in HT assays that assessed CYP inhibition, inhibition of dofetilide binding, and cellular toxicity.

The potential for drugs and candidates to mediate drug-drug interactions (DDI) through perturbation of clearance mechanisms for other drug substances was assessed by measuring the inhibition of CYP2D6 and CYP3A4 in HT screening assays. These assays were conducted with HLM and contained control substrates for these CYPs together with compounds of interest. Data was recorded as percent inhibition (\% inh) of reference control substrate metabolism and was interpreted as follows with regard to risk for DDI: $\%$ inh $\leq$ 25 as "low risk", $25<\%$ inh $\leq 75$ as "moderate risk", and \% inh > 75 as "high risk" for both CYP2D6 and CYP3A4 substrates (see Supporting Information for tabulated data). Of the 99 drugs and 101 candidates for which we obtained experimental CYP inhibition data, over $80 \%$ of compounds tested were classified as low risk for causing DDI through either CYP2D6 or CYP3A4 inhibition (Figure 7), and no statistically significant differences were found between drugs and candidates. The data obtained suggests that most of these drugs and candidates occupy desirable, low risk space for DDI.

Blocking of the potassium hERG channel may result in prolongation of the QT interval of cardiac rhythm. Inhibition of dofetilide binding to the hERG channel has been used as a surrogate to assess potential blockade of this channel (30). In vitro dofetilide inhibition data for the drugs (90 out of 119) and the candidates (88 out of 108) was obtained by measuring competitive binding to the dofetilide binding site in HEK-hERG membrane homogenates (See Supporting Information for tabulated results). The output examined was percent inhibition ( $\%$ inh) of dofetilide binding, where the higher the value, the higher the risk of interference with the hERG 
A)

2D6

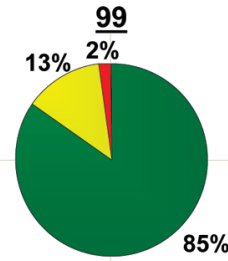

B)

3A4

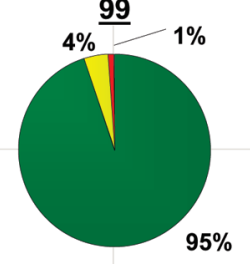

Drugs

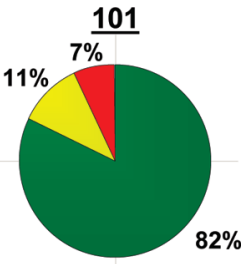

101

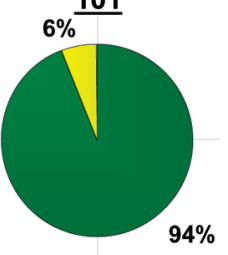

Candidates
Figure 7. Drug and candidate distribution of potential drug-drug interactions (DDI) for (A) CYP2D6 and (B) CYP3A4. Results are displayed as percent inhibition ( $\%$ inh) of reference control substrate metabolism and are interpreted as follows with regard to risk for DDI: $\%$ inh $\leq 25$ (low risk, green), $25<\%$ inh $\leq 75$ (moderate risk, yellow), $\%$ inh $>75$ (high risk, red) for both CYP2D6 and CYP3A4 substrates.

cardiac ion channel. Results were classified as follows: \% inh $<15$ as low risk, $15 \leq \%$ inh $\leq 50$ as moderate risk, and $\%$ inh $>50$ as high risk. Analysis of the data revealed that $57 \%$ of the drugs and $43 \%$ of the candidates had low binding to the dofetilide site in the hERG channel (Figure 8A). While the difference in dofetilide inhibition was not statistically significant $(p=0.1003)$ between drugs and candidates, we hypothesized that the larger percentage of compounds in the candidate set with moderate to high risk was due in part to their higher $\mathrm{C} \log \mathrm{P}$. When the data were grouped into two $\mathrm{Clog} \mathrm{P}$ bins, $\mathrm{C} \log \mathrm{P}>3$ and $\mathrm{C} \log \mathrm{P} \leq 3$ (Figure $8 \mathrm{~B}$ ), a dramatic trend was observed for both sets of compounds: over $70 \%$ of the compounds with $\mathrm{Clog} \mathrm{P} \leq 3$ exhibited low binding to the dofetilide site in the hERG channel (low risk) and greater than $70 \%$ of compounds with $\mathrm{Clog} P>$ 3 had moderate to high binding to the dofetilide site in the hERG channel (moderate to high risk). There was a larger proportion of compounds in the higher $\mathrm{Clog} P$ space $(C \log P>3)$ for the candidate set as opposed to the drug set, supporting the hypothesis mentioned above regarding lipophilicity (Figure 8B). The drug set was further partitioned by $\mathrm{p} K_{\mathrm{a}}$, to determine whether incorporation of an additional physicochemical property would increase the odds of identifying the higher risk compounds (Figure 9). When ClogP was combined with $\mathrm{p} K_{\mathrm{a}}$, an increased refinement in identifying compounds with potential hERG liability was indeed observed. The drug subset exhibiting both high $C \log P$ and elevated $\mathrm{p} K_{\mathrm{a}}$ had a significantly increased percentage of compounds with high inhibition of dofetilide binding ( $>50 \%$ inh) compared with those with low $C \log P$ and low $\mathrm{p} K_{\mathrm{a}}$. Thus, both $\mathrm{ClogP}$ and $\mathrm{p} K_{\mathrm{a}}$ can be used prospectively in design
A)

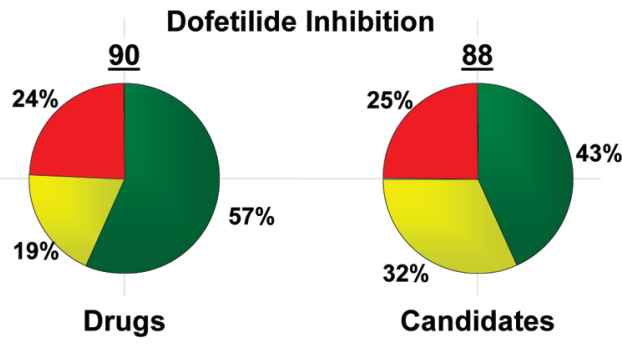

B)
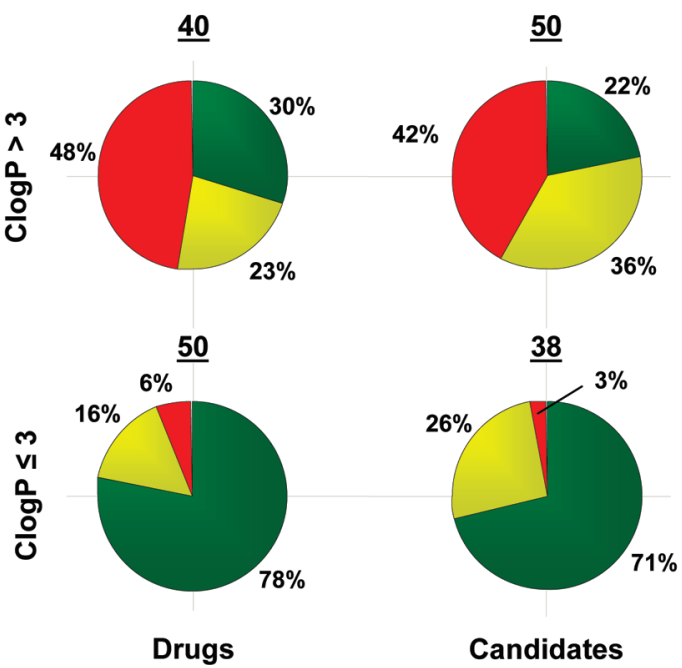

Figure 8. Analysis of percent inhibition (\% inh) of dofetilide binding for drugs and candidates. The pie charts display color-coded potential risk of hERG interaction: low risk ( $\%$ inh $\leq 15$, green $)$, moderate risk $(15<\%$ inh $\leq 50 \%$, yellow $)$, high risk $(\%$ inh $>50$, red). (A) Distribution of binned dofetilide binding for both drugs and candidates. (B) The binned dofetilide binding of drugs and candidates was further grouped by $\mathrm{C} \log \mathrm{P}$ : ClogP $\leq 3$ and $\mathrm{Clog} \mathrm{P}>3$.

to improve the odds of identifying compounds with reduced risk of binding to the hERG channel (Figure 9).

In addition to the two in vitro safety assays described above, we examined cell viability outcomes for the drugs and the candidates. The data was generated using a transformed human liver epithelial cell line, and the output was reported as $\mathrm{IC}_{50}$ values in $\mu \mathrm{M}$, where the lower the $\mathrm{IC}_{50}$ value, the greater the cell toxicity. The $\mathrm{IC}_{50}$ 's were classified as follows: transformed human liver epithelial cell viability (THLE Cv) $\leq 100 \mu \mathrm{M}$ (low cell viability) and THLE Cv $>100 \mu \mathrm{M}$ (high cell viability).

Of the 119 drugs and 108 candidates, we were able to obtain THLE $\mathrm{Cv} \mathrm{IC} 50$ 's on 81 and 102 compounds, respectively. The cell viability $\mathrm{IC}_{50}$ values for the drug set ranged from $18.5 \mu \mathrm{M}$ to greater than $300 \mu \mathrm{M}$, with a majority of them $(79 \%)$ having THLE Cv $>100 \mu \mathrm{M}$ (Figure 10). Applying the same THLE Cv cutoff of $>100 \mu \mathrm{M}, 71 \%$ of the candidates showed high cell viability, statistically the same as the drug set ( $p=$ 0.1924). Based on these criteria, most of these drugs 

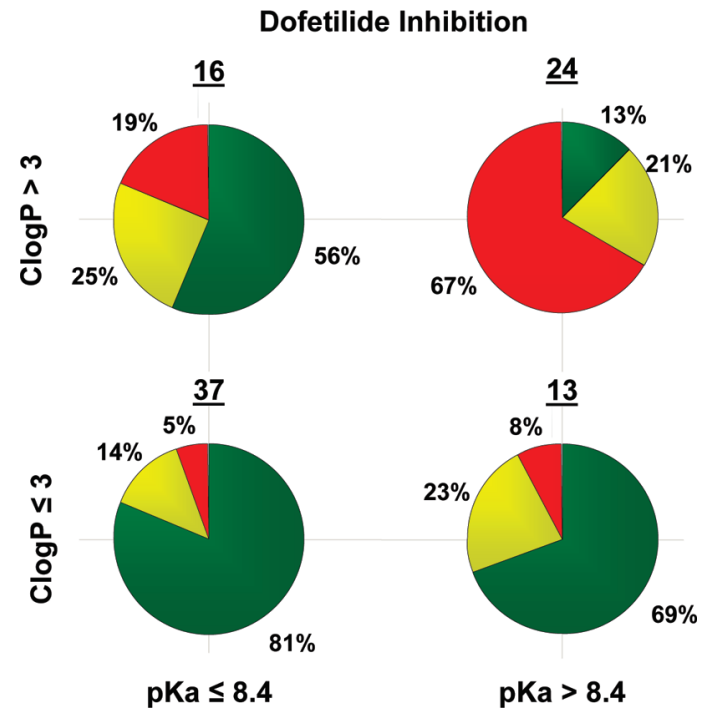

Figure 9. Dofetilide inhibition data for the drug set versus ClogP and $\mathrm{p} K_{\mathrm{a}}$. Drugs with both higher $C \operatorname{logP}$ and higher $\mathrm{p} K_{\mathrm{a}}$ had a significantly increased percentage of compounds with high binding ( $>50 \%$ inh) to the dofetilide site compared with those with low $\mathrm{C} \log \mathrm{P}$ and low $\mathrm{p} K_{\mathrm{a}}$. The pie charts are color-coded by potential risk of hERG channel blockade: low risk ( $\%$ inh $\leq 15$, green $)$, moderate risk $(15<\%$ inh $\leq 50$, yellow $)$, high risk $(\%$ inh $>50$, red $)$.

and candidates occupy desirable, low risk cellular toxicity space. Lipophilicity is a dominant factor in both promiscuity (6) and the 3 and 75 relative risk factors (18); therefore we also analyzed the THLE Cv data by segregating it into two ClogP bins, high lipophilicity $(\mathrm{C} \log \mathrm{P}>3)$ and low lipophilicity $(\mathrm{Clog} \mathrm{P} \leq 3)$ (Figure 10B). Across the drugs and the candidates, a higher percentage of compounds $(>90 \%)$ in the low lipophilicity bin $(\mathrm{Clog} \mathrm{P} \leq 3)$ had high cell viability (THLE $\mathrm{Cv}>100 \mu \mathrm{M}$ ) in comparison to compounds $(62 \%$ or $56 \%)$ in the high lipophilicity bin $(\mathrm{ClogP}>3)$.

The drug set was further analyzed by using two TPSA bins, TPSA $\leq 75$ and TPSA $>75$, to determine whether further improvement in identification of the more risky compounds could be achieved based on the 3 and 75 RRFs. Using these two TPSA bins, no statistical difference or improved odds in the identification of compounds with poor THLE Cv was achieved (data not shown). However, when $\mathrm{Clog} P$ was combined with $\mathrm{MW}$ (median value) for the drug set, an enhanced segregation of compounds with in vitro toxicity was observed (Figure 11). This data reinforces that lipophilicity is not the only influencing factor in cell viability outcome, a measure of potential in vivo toxicity.

\section{Alignment of Safety Attributes}

Independent examination of each of the safety properties (DDI, dofetilide binding, and cellular toxicity) suggested that drugs on average are comparable to the candidates, with no statistically significant differences. To assess the safety alignment of drugs and candidates,
A)

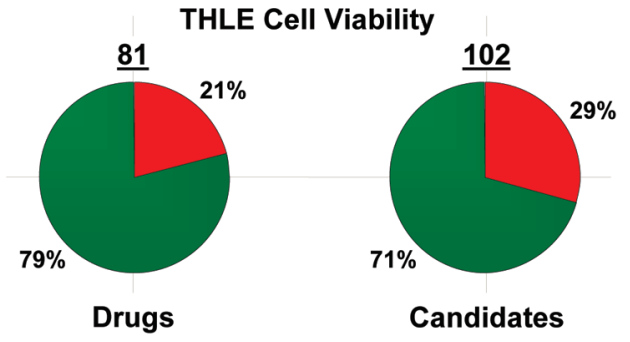

B)
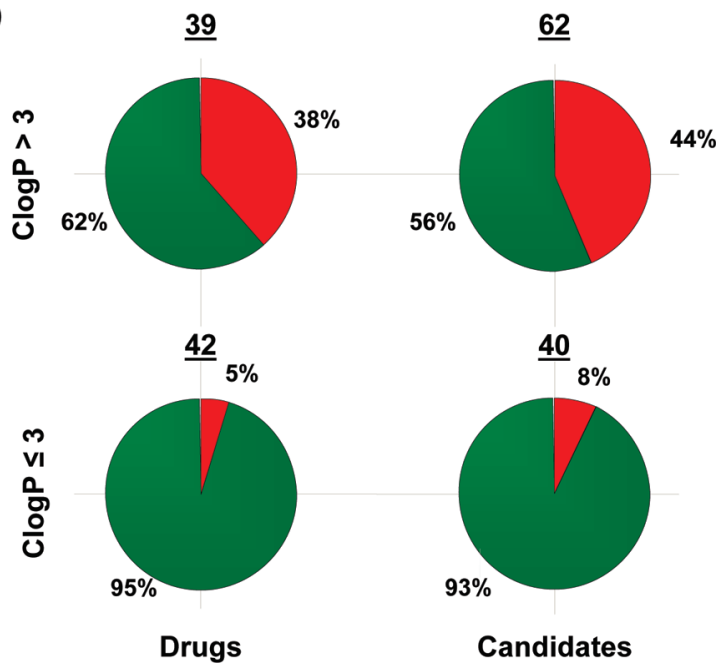

Figure 10. Distribution of THLE Cv of drugs and candidates as measured by an ATP depletion assay. Results are color-coded by high cell viability $\left(\mathrm{IC}_{50}>100 \mu \mathrm{M}\right.$, green) and low cell viability ( $\mathrm{IC}_{50} \leq 100 \mu \mathrm{M}$, red). (A) Overall distribution of THLE $\mathrm{Cv}$ for the drug and candidate set. (B) Segregation of THLE Cv data into high and low lipophilicity bins.

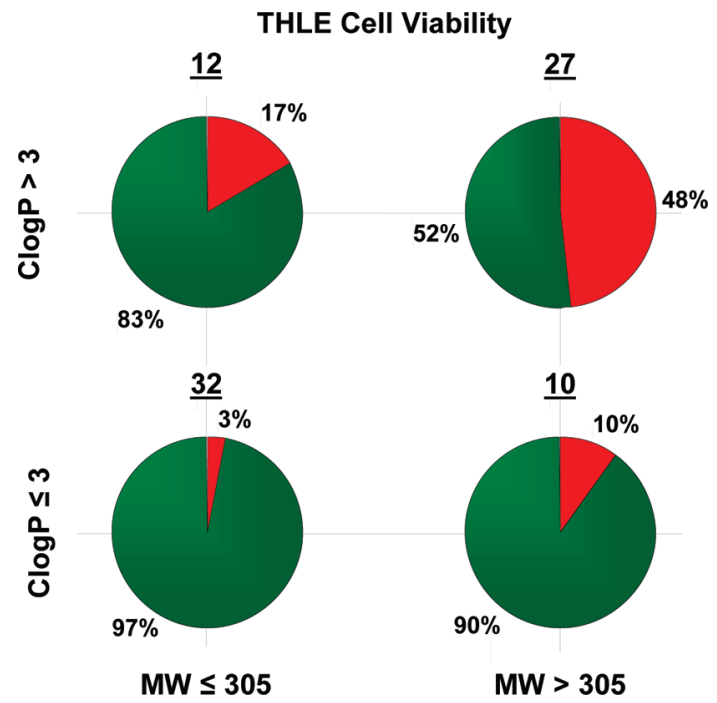

Figure 11. Partitioning of THLE Cv data for the drug set by ClogP and MW. The charts are color-coded by high cell viability $\left(\mathrm{IC}_{50}>100 \mu \mathrm{M}\right.$, green) and low cell viability $\left(\mathrm{IC}_{50} \leq 100 \mu \mathrm{M}\right.$, red $)$.

we characterized each compound by the number of most desirable safety attributes possessed according to the 
following criteria: low DDI with both 2D6 and 3A4 substrates ( $\%$ inh $\leq 25 \%)$, low dofetilide binding $(\%$ inh $\leq 15 \%)$, and high THLE CV $\left(\mathrm{IC}_{50}>100 \mu \mathrm{M}\right)$. A count of $0-3$ safety attributes was possible for each molecule as shown in Figure 12. The drug set was heavily populated with compounds exhibiting full alignment of the three safety attributes $(34 / 77,44 \%)$. In comparison with the drug set, the candidate set showed a trend for fewer compounds $(28 / 84 ; 33 \%)$ with fully aligned desired attributes in one molecule. The difference between the alignment distributions of the drug and

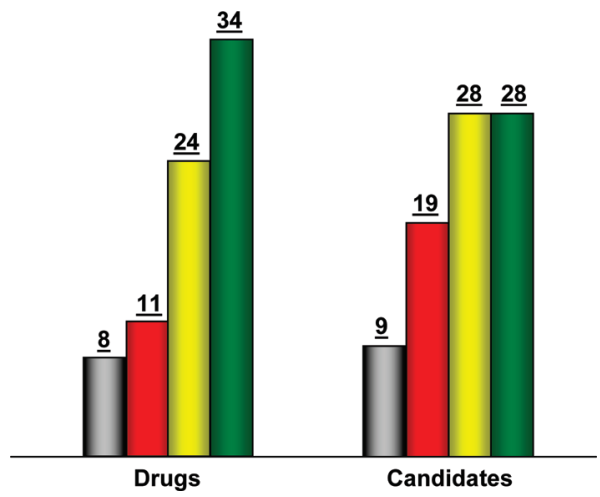

Figure 12. Safety attribute alignments of drugs and candidates, colorcoded by the number of times a molecule achieved low DDI for both $2 \mathrm{D} 6$ and $3 \mathrm{~A} 4$, low dofetilide binding, or high THLE Cv: full alignment (3/3) attributes (green), 2/3 attributes (yellow), 1/3 attributes (red), $0 / 3$ attributes (gray). candidate sets was not statistically significant ( $p=$ 0.4230). The highlighted safety assays may increase the probability of success; however, further improvement in the positive predictive values of new assays will be essential to enable medicinal chemists to more rapidly identify best in class molecules.

Lastly, we examined the drug and candidate sets for full alignment of both ADME and safety attributes using the criteria outlined above. For the 52 drugs with all six measured end points, 18 compounds $(35 \%)$ possessed full alignment (6/6) of the desirable attributes (Figure 13). Consistent with the earlier analysis of the ADME and safety attribute alignment, the candidate set had fewer fully aligned attributes, 9 out of 62 compounds $(14 \%)$. Full alignment of these in vitro parameters is a potential key distinguishing factor for the success of drugs with favorable therapeutic indices.

\section{Conclusion}

There are numerous design parameters that medicinal chemists can follow in the drug discovery process and defining the parameters that increase the probability of identifying best in class molecules is of critical importance. Understanding the fundamental relationships between physicochemical properties and in vitro and in vivo outcomes is the foundation required to prospectively design compounds with an overall favorable profile. In our quest to further build this understanding in

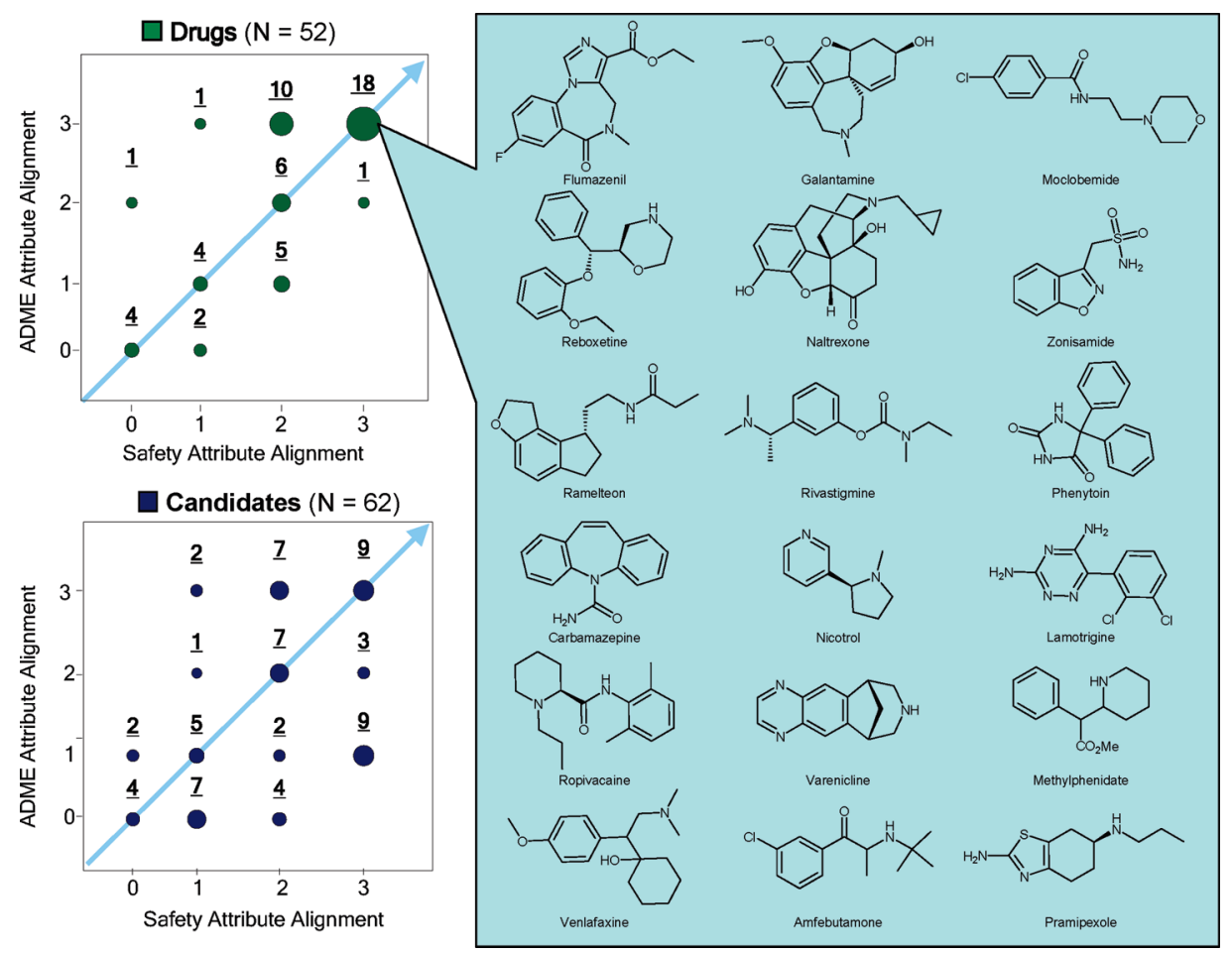

Figure 13. Plots of ADME attribute alignment vs safety attribute alignment for the drug and candidate sets. The drugs with full alignment (6/6) of ADME and safety attributes are exemplified. 


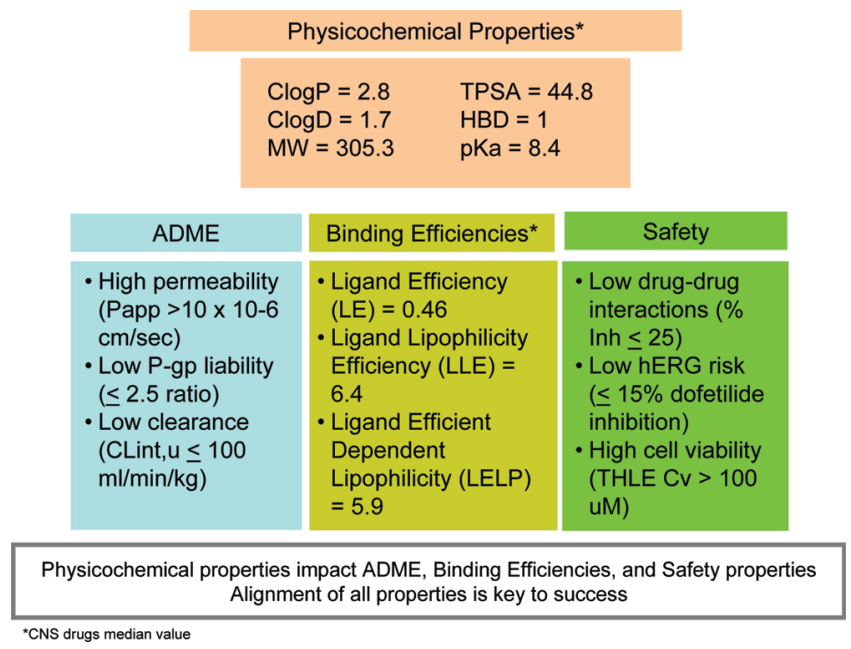

Figure 14. Drug optimum values.

the CNS chemical space, we undertook a thorough and detailed analysis of the physicochemical properties, in vitro ADME attributes, primary pharmacology binding efficiencies, and in vitro safety end points for 119 drugs and 108 candidates.

We examined six fundamental physicochemical properties associated with these sets of compounds: ClogP, ClogD, MW, TPSA, HBD, and $\mathrm{p} K_{\mathrm{a}}$. The CNS drug space defined by these six physicochemical properties is quite broad, but our scholarship points to optimum ranges for each of these properties. Based on the drug set, median values were found to be $C \log P=2.8$, $\mathrm{Clog} \mathrm{D}=1.7, \mathrm{MW}=305.3 \mathrm{Da}, \mathrm{TPSA}=44.8 \AA^{2}, \mathrm{HBD}=$ 1 , and $\mathrm{p} K_{\mathrm{a}}=8.4$ (Figure 14). Data generated in-house in ADME high-throughput assays reinforced that the majority of CNS drugs are highly permeable (MDCK $P_{\text {app }}>10 \times 10^{-6} \mathrm{~cm} / \mathrm{s}$ ), with little or no P-gp efflux liability (P-gp ratios $\leq 2.5$ ), and with low to moderate clearance rates $\left(\mathrm{CL}_{\mathrm{int}, \mathrm{u}} \leq 100 \mathrm{~mL} /(\mathrm{min} \cdot \mathrm{kg})\right)$. Based on reported binding affinities to the primary pharmacological target, we also determined that $>90 \%$ of the drugs have LE and LLE values $>0.37$ and $>3.3$, respectively, and LELP $\leq 11.9$ (10th percentile of these parameters). Median LE, LLE, and LELP values for the marketed drug set were $0.46,6.4$, and 5.9, respectively, with some CNS drugs displaying remarkable binding efficiencies ( $\mathrm{LE}>0.6$ and LLE > 7.9). In general, CNS drugs showed minimal drug-drug interactions (measured by CYP3A4 and CYP2D6 inhibition) and hERG liability (measured by inhibition of dofetilide binding) as determined in high-throughput assays inhouse. Our analysis reinforced that binding to the dofetilide site at the hERG channel was influenced by lipophilicity and basicity. Finally, the majority of marketed drugs tested in-house in an in vitro safety assay utilizing a THLE cell line showed good cell viability (low toxicity) at high concentrations $\left(\mathrm{IC}_{50}>100 \mu \mathrm{M}\right)$. This analysis further suggested that lipophilicity and molecular weight are factors influencing cell viability outcomes, with low cellular toxicity $(>100 \mu \mathrm{M})$ in the THLE $\mathrm{Cv}$ assay and low lipophilicity $(\mathrm{Clog} \mathrm{P} \leq 3)$ potentially improving the odds of identifying CNS compounds that may become drugs.

As part of this analysis, we included a set of Pfizer candidates to understand potential similarities and differences between compounds that successfully reached the market and the candidate set. This candidate set included both active and discontinued compounds and excluded Pfizer candidates that had reached drug status, such as varenicline, pregablin, ziprasidone, and sertraline. The candidates showed significant differences in the median values associated with $\mathrm{ClogP}$ and $\mathrm{MW}$ in comparison to the marketed drug set. Median values for these two properties were higher for the candidates in comparison to the drugs (ClogP median value of $3.3 \mathrm{vs}$ 2.8 and MW median value of $360.4 \mathrm{vs} 305.3$ ). The higher ClogP and MW median values associated with the candidate set had an impact on other attributes such as ADME (permeability, P-gp efflux, and clearance) and LELP properties, resulting in less favorable profiles for the candidates in comparison to drugs. Unlike LE and LLE, LELP median values were statistically different for the drugs vs candidates (5.9 vs 7.0) suggesting that both size and lipophilicity may be important to enable the identification of compounds with optimal binding efficiencies. In comparison to the candidates, the drug set showed a greater proportion of compounds with aligned ADME and safety attributes in one molecule, suggesting that holistic alignment of attributes is a key to increased probability of success in the discovery of drugs. These results are not surprising, because the CNS drug set is comprised of molecules that have survived to the market and thus does not provide a perspective on the properties of compounds that were not selected along the way as the marketed drugs were being discovered and developed. In addition, it is important to reinforce that the candidate set included in this analysis spans nearly 20 years of research. During this period of time, our understanding of good physicochemical property space has significantly improved, and the availability and use of high-throughput in vitro ADME and safety assays to characterize a large number of compounds has become routine. Nevertheless, our analysis suggests that for centrally acting drugs, there may be a need to design compounds with further reduced $\mathrm{ClogP}, \mathrm{MW}$, or both to better match the corresponding properties in the marketed drug set.

As demonstrated by our analysis, physicochemical properties, ADME attributes, target binding efficiencies, and safety in vitro attributes are all important parameters to enable better candidate selections, saving significant time and resource in the drug development 
process. In particular, we were interested in understanding key physicochemical properties associated with the CNS drug space to better inform the design of future drug candidates with the desired druglike and safety properties. These physicochemical properties (ClogP, ClogD, MW, TPSA, HBD, and $\mathrm{p} K_{\mathrm{a}}$ ) impact a variety of in vitro end points that medicinal chemists strive to align, such as ADME end points (permeability $P_{\text {app }}$, P-gp efflux, and metabolic stability), binding efficiencies, and safety end points. Lipophilicity $(\mathrm{C} \log \mathrm{P})$ continues to rise to the top as an important physicochemical property and recent disclosures, for example, the 3 and 75 relative risk factors by Pfizer scientists, reinforce the role of this property in safety outcomes. However, utilizing one or two properties or attributes in the design may be too restrictive and may not fully enable the alignment of metabolic stability, permeability, CNS penetration, and safety properties in one molecule. Hence, we believe that a favorable approach will involve the use of a multiparametric optimization that takes into consideration optimum ranges associated with all six physicochemical parameters in CNS drugs to balance these properties and expand the design space. Ultimately, our goal is to move our medicinal chemistry design to a space of higher probability of success to increase speed in the identification of candidates that survive to test hypotheses in the clinic and that have a good chance of becoming marketed CNS agents.

\section{Methods}

\section{Data Collection}

Marketed CNS Drugs. The drug set used in this study includes 119 centrally active drugs. This group of 119 drugs serves as a representative set of CNS drugs and was not meant to be all-inclusive. To the best of our knowledge all compounds in the drug set could be used as oral agents. Several drugs with mixed reports of being centrally active were omitted from the data set as well as some combination drugs. We either obtained drug substances from the Pfizer internal collection or purchased them from an external vendor in pure form. All samples were handled in accordance with Pfizer and the Federal Drug Enforcement Administration (DEA) polices. A complete list of the drugs used in the analysis appears in Table 1.

Pfizer CNS Candidates. The candidates included in our analysis consisted of all 108 compounds that met the appropriate preclinical profile to advance into regulatory safety studies and, if appropriate, clinical studies. Marketed Pfizer candidates, such as varenicline, pregabalin, and ziprasidone, were not included in the candidate set, but in the drug set. Solid samples were obtained from the Pfizer internal collection and
Table 1. Marketed Drug Set by Name

\begin{tabular}{|c|c|c|}
\hline \multicolumn{3}{|c|}{ Central Nervous System Drugs } \\
\hline acamprosate & fluvoxamine & propoxyphene \\
\hline alprazolam & gabapentin & quazepam \\
\hline amfebutamone & galantamine & quetiapine \\
\hline amisulpride & haloperidol & ramelteon \\
\hline amphetamine & hydrocodone & rasagiline \\
\hline aniracetam & indeloxazine & reboxetine \\
\hline apomorphine & lamotrigine & remifentanil \\
\hline aprepitant & levetiracetam & riluzole \\
\hline aripiprazole & levomethadyl & rimonabant \\
\hline atomoxetine & lofexidine & risperidone \\
\hline bromazepam & lorazepam & rivastigmine \\
\hline bromocriptine & meprobamate & ropinirole \\
\hline brotizolam & methylphenidate & ropivacaine \\
\hline budipine & metoclopramide & selegiline \\
\hline buprenorphine & midazolam & sertindole \\
\hline buspirone & milnacipran & sertraline \\
\hline cabergoline & minaprine & sinequan \\
\hline caffeine & mirtazapine & sulpiride \\
\hline carbamazepine & moclobemide & tacrine \\
\hline carisoprodol & modafinil & talipexole \\
\hline chlorpromazine & morphine & terguride \\
\hline citalopram & nalmefene & thiopental \\
\hline clomipramine & naltrexone & tiagabine \\
\hline clonazepam & nemonapride & tianeptine \\
\hline clozapine & nicergoline & topiramate \\
\hline cyclobenzaprine & nicotrol & tramadol \\
\hline dexmethylphenidate & nimodipine & trazodone \\
\hline dextropropoxyphene & nortriptyline & tropisetron \\
\hline diazepam & olanzapine & valproic acid \\
\hline donepezil & oxcarbazepine & varenicline \\
\hline dronabinol & oxycodone & venlafaxine \\
\hline duloxetine & paliperidone & verapamil \\
\hline eletriptan & paroxetine & vigabatrin \\
\hline escitalopram & pergolide & vinpocetine \\
\hline eszopiclone & perospirone & zaleplon \\
\hline ethosuximide & phenytoin & ziprasidone \\
\hline felbamate & piracetam & zolpidem \\
\hline fentanyl & pramipexole & zonisamide \\
\hline flumazenil & pregabalin & zotepine \\
\hline fluoxetine & propofol & \\
\hline
\end{tabular}

handled in accordance with Pfizer policies. The candidate set utilized in this analysis represents 40 unique mechanisms that span nearly two decades of research from Pfizer (including CNS candidates obtained via companies acquired by Pfizer). To the best of our knowledge, all of these candidates targeted the oral dosing route of administration. The list of target families and number of candidates from each target family for the candidate set appears in Table 2.

Physicochemical Property Data. This analysis included calculated partition coefficient $(\mathrm{ClogP})$, 
Table 2. Candidates by Target

\begin{tabular}{rll}
\hline candidate count & target mechanism & \multicolumn{1}{c}{ target family } \\
\hline 13 & enzymes & enzymes \\
4 & cannabinoids & GPCR \\
13 & dopaminergic GPCR & GPCR \\
2 & histaminergic GPCR & GPCR \\
2 & muscarinic & GPCR \\
4 & opioids & GPCR \\
13 & peptide GPCR & GPCR \\
16 & serotonergic GPCR & GPCR \\
6 & calcium channel & ion channels \\
11 & GABA receptors & ligand-gated ion channel \\
5 & glutamate receptors & ligand-gated ion channel \\
8 & nicotinic & ligand-gated ion channel \\
11 & reuptake inhibitors & transporters \\
\hline
\end{tabular}

calculated distribution coefficient at $\mathrm{pH}=7.4(\mathrm{ClogD})$, molecular weight (MW), topological polar surface area (TPSA) (19), the number of hydrogen bond donors (HBD), and the calculated most basic center $\left(\mathrm{p} K_{\mathrm{a}}\right)$. Calculated physicochemical properties were obtained using standard commercial packages: Biobyte for ClogP calculations; ACD laboratories for $\mathrm{ClogD}$ at $\mathrm{pH} 7.4$ and the most basic $\mathrm{p} K_{\mathrm{a}}$. TPSA was calculated using the method developed by Ertl (19).

Statistical analyses were carried out using SAS JMP 7 statistical software (33), and the data was visualized with JMP or Spotfire Decision Site (34). Differences between drugs and candidates were statistically examined by Student's $t$ test for continuous variables (Figure 1 and Figure 5) or by likelihood ratio $\chi^{2}$ test of logistic regressions for ordinal classifications (Figures 2, 3, 4, 7, 8, 10, and 12). Statistical significance is defined by a $p$-value of less than 0.05 .

ADME Data. Data on the following in vitro ADME properties were generated in-house utilizing the corresponding HT assays: (a) passive apparent permeability $\left(P_{\text {app }}\right)$, assayed utilizing the Madin-Darby canine kidney (MDCK) cell line, (b) P-glycoprotein (P-gp) efflux liability, assessed via an assay utilizing the MDR1-MDCK cell line, an MDCK line stably transfected with the MDR1 gene that expresses a functionally active P-gp, and (c) metabolic stability, expressed as unbound intrinsic clearance $\left(\mathrm{CL}_{\mathrm{int}, \mathrm{u}}\right)$ calculated according to eq 2 (22), using the measured intrinsic clearance $\left(\mathrm{CL}_{\text {int,app }}\right)$, obtained via an in vitro, HT human liver microsome assay and an in silico model for free microsome fraction $\left(\mathrm{cF}_{\mathrm{u}, \mathrm{mic}}\right)$.

Compounds included in the above studies were handled as $30 \mathrm{mM}$ stock solutions, which were generated, dispensed, and checked for purity by Pfizer's internal sample bank, and subsequently assayed in the ADME assays. The ADME assays were performed via reported methods as described previously for
MDCK $P_{\text {app }}(14)$, MDR-MDCK P-gp (14), and metabolic stability $(14,15,22,35)$.

Potency Data. Potency data was defined as in vitro $K_{\mathrm{i}}$ or $\mathrm{IC}_{50}$ values reported in nanomolar at the defined primary pharmacology target. In vitro binding data were obtained from public databases (Prous, Wikipedia, DrugBank, IDDB, PharmaPendium, PharmaMatrix, Thomson, and Goodman and Gilman) for drugs and from in-house databases for the candidates. For drugs and candidates with multiple pharmacology profiles, the most active target was used in ligand efficiency analyses $(16,17)$. Drugs with inconsistent potency values throughout the literature were not included in the ligand efficiency analyses. Rodent in vitro binding end points were used when human in vitro binding data were not available. See Supporting Information for details.

Safety Data. Data for the following in vitro safety end points were generated in house via HT assays: (a) drug-drug interactions, assessed via inhibition of cytochrome P450 (CYPs) monooxygenase enzymes, in particular, 3A4 and 2D6 (28); (b) hERG liability, assessed via inhibition of dofetilide binding (30) as a surrogate indicator of hERG potassium ion channel effects (blocking of hERG may result in prolongation of the QT interval of cardiac rhythm) (29), and (c) cellular toxicity, measured as activity in an in vitro cellular toxicity assay as a surrogate for acute in vivo toxicity (32). Assays were performed via reported methods as described previously for DDI (28) and dofetilide binding (30). The cellular toxicity data was generated using a transformed human liver epithelia cell line where ATP levels were detected using a bioluminescent end point. This assay utilized luciferase to catalyze the formation of light from ATP and luciferin (eq 5).

$$
\begin{aligned}
& \text { ATP }+ \text { Luciferin }+\mathrm{O}_{2} \longrightarrow \text { Luciferase } \\
& \mathrm{AMP}+\text { pyrophosphate }+\mathrm{CO}_{2}
\end{aligned}
$$

Transformed Human Liver Epithelial Cell Viability Assay. THLE-2 (transformed human liver epithelial) cells were obtained from ATCC (CRL-2706 or CRL10149) and cultured according to ATCC recommendations. Media consisted of basal medium (BEGM Bullet Kit, Lonza Cat. no. CC-3170), supplemented with 10\% fetal bovine serum (Sigma Cat. no. F4135), $2.5 \mathrm{ng} / \mathrm{L}$ hEFG (BD Biosciences Cat. no. 356052), and $700 \mathrm{ng} / \mathrm{L}$ phosphoethanolamine (Sigma Cat. no. p-0503). Cells were cultured in T175 human fibronectin/collagen/ bovine serum albumin-coated flasks. For each experiment, cells were plated onto 384 plates (human fibronectin/collagen/bovine serum albumin-coated 384, 
custom order, BD Biosciences Cat. no. 359298) at a cell density of $2.5 \times 10^{3} /$ well in a total medium volume of $25 \mu \mathrm{L} /$ well. Plates were incubated for $24 \mathrm{~h}$ at $37^{\circ} \mathrm{C}, 5 \%$ $\mathrm{CO}_{2}$.

Compound test plates were prepared using a 10 dose, 2.0-fold dilution scheme with a final assay concentration range from 300 to $0.058 \mu \mathrm{M}$. All compounds were initially solubilized in $100 \%$ DMSO. This dosing scheme contains 32 compounds per plate. Stock plates were prepared by aliquoting $1 \mu \mathrm{L}$ of $100 \times$ compound/ well $(30-0.058 \mathrm{mM})$. The plates were prepared for dosing by adding $99 \mu \mathrm{L}$ of cell culture media and mixing. Test compounds were added to cell culture plates by aspirating the overnight culture media and replacing it with $25 \mu \mathrm{L} /$ well of media containing test compound. The final concentration of DMSO in each well was $1.0 \%$.

Following a $72 \mathrm{~h}$ exposure to test compounds, cell viability in each well was determined by assessing the depletion of cellular ATP using the Lonza Vialight Plus Cell Proliferation/Cytoxicity Kit (Lonza cat. no. LT07-119) according to the manufacturer's protocol. The ATP concentration was determined by reading luminescence using a Wallac EnVision plate reader (PerkinElmer, Waltham, MA). The percent of viable cells relative to no-drug treated controls was determined for each well. Final data output was a calculated $\mathrm{IC}_{50}$ value describing the dose projected to kill $50 \%$ of the cells following a $72 \mathrm{~h}$ exposure.

\section{Acknowledgment}

The authors thank Joe Brady, Mike Banker, Brian Bronk, David Gray, Chris Helal, Theodore Liston, Spiros Liras, Scot Mente, Ronald Obach, William Pennie, and Shibing Deng for helpful discussions. The authors thank Eric Benvenuti, Chi-Kin Chan, Karen Erikson, James Federico, Carrie Funk, Jeffery Osowski, Michael Pacheco, Caroline Proulx-Lafrance, Nora Wallace, Mitchell Walter, Michael West, and Carrie Whitney-Pickett for compound management or data collection. The authors thank Katherine Brighty, George Chang, Stefan Steyn, Christopher O'Donnell, and David Price for their insightful comments on this manuscript.

\section{Supporting Information Available}

The following drug and candidate set data in tabulated form: Pub-Name, Set, Target Mechanism, MW, CLogP, TPSA, CLogD, HBD, $K_{\mathrm{a}}$, LE, LLE, LELP, Papp Op, Papp Mean, Papp SD, Papp CV, Papp SEM, PappUpp95, Papp Low95, Papp N, Pgp OP, Pgp Mean, Pgp SD, Pgp CV, Pgp SEM, Pgp Upp95, Pgp Low95, Pgp N, cFu,mic, CLIA OP, CLIA Mean, CLIA SD, CLIA CV, CLIA SEM, CLIA Upp95, CLIA Low95, CLIA N, CLIu, 2D6 Inh (\%@3uM) OP, 2D6 Inh (\%@3uM) Mean, 2D6 Inh (\%@3uM) SD, 2D6 Inh (\% @3uM)CV, 2D6Inh (\%@3uM), SEM 2D6Inh (\%@3uM), Upp95 2D6 Inh (\%@3uM), Low95 2D6 Inh (\%@3uM), N
3A4 Inh (\%@3uM) OP, 3A4 Inh (\%@3uM), Mean 3A4 Inh (\%@3uM), SD 3A4 Inh (\%@3uM), CV 3A4 Inh (\% $(3 \mathrm{uM})$, SEM 3A4 Inh (\%@3uM) Upp95, 3A4 Inh (\%@3uM), Low95 3A4 Inh (\%@3uM), TotCnt 3A4 Inh (\%@3uM), N Dof Inh (\%@10uM), Mean Dof Inh (\% ( $10 \mathrm{uM})$, SD Dof Inh $(\% @ 10 \mathrm{uM})$, CV Dof Inh ( $\%$ ( $10 \mathrm{uM})$, SEM Dof Inh $(\% @ 10 \mathrm{uM})$, Upp95 Dof Inh (\% @ 10uM), Low95 Dof Inh (\%@10uM), (\%@10uM), THLE $\mathrm{Cv}_{\mathrm{IC}}{ }_{50} \mathrm{OP}, \mathrm{THLE} \mathrm{Cv} \mathrm{IC}_{50}$ Mean, THLE Cv IC 50 SD, THLE $\mathrm{Cv} \mathrm{IC}_{50} \mathrm{CV}$, THLE Cv $\mathrm{IC}_{50}$ SEM, THLE Cv IC 50 Upp95, THLE Cv $\mathrm{IC}_{50}$ Low95, THLE Cv $\mathrm{IC}_{50} \mathrm{~N}$. This material is available free of charge via the Internet at http://pubs.acs.org.

\section{Author Information}

\section{Corresponding Author}

* Mailing address: Pfizer PharmaTherapeutics Research and Development, 558 Eastern Point Road, Groton, CT 06340-5159. Tel: 860-715-4059. Fax: 860-686-6052. E-mail: travis.t.wager@ pfizer.com.

Author Contributions

${ }^{\dagger}$ These authors contributed equally to this work.

\section{References}

1. Kola, I., and Landis, J. (2004) Opinion: Can the pharmaceutical industry reduce attrition rates? Nat Rev. Drug Discovery $3,711-716$.

2. O'Shea, R., and Moser, H. E. (2008) Physicochemical properties of antibacterial compounds: Implications for drug discovery. J. Med. Chem. 51, 2871-2878.

3. Leeson Paul, D., and Davis Andrew, M. (2004) Timerelated differences in the physical property profiles of oral drugs. J. Med. Chem. 47, 6338-6348.

4. Veber, D. F., Johnson, S. R., Cheng, H.-Y., Smith, B. R., Ward, K. W., and Kopple, K. D. (2002) Molecular properties that influence the oral bioavailability of drug candidates. J. Med. Chem. 45, 2615-2623.

5. Proudfoot, J. R. (2005) The evolution of synthetic oral drug properties. Bioorg. Med. Chem. Lett. 15, 1087-1090.

6. Leeson Paul, D., and Springthorpe, B. (2007) The influence of drug-like concepts on decision-making in medicinal chemistry. Nat. Rev. Drug Discovery 6, 881-890.

7. Gleeson, M. P. (2008) Generation of a set of simple, interpretable ADMET rules of thumb. J. Med. Chem. 51, 817-834.

8. Pajouhesh, H., and Lenz, G. R. (2005) Medicinal chemical properties of successful central nervous system drugs. NeuroRX 2, 541-553.

9. Lipinski, C. A. (2005) Filtering in drug discovery. Annu. Rep. Comput. Chem. 1, 155-168.

10. Hitchcock, S. A., and Pennington, L. D. (2006) Structure- brain exposure relationships. J. Med. Chem. 49, 7559-7583.

11. Leeson, P. D., Davis, A. M., and Steele, J. (2004) Druglike properties: Guiding principles for design - or chemical prejudice? Drug Discovery Today: Technol 1, 189-195. 
12. Abraham, M. H., Ibrahim, A., Zissimos, A. M., Zhao, Y. H., Comer, J., and Reynolds, D. P. (2002) Application of hydrogen bonding calculations in property based drug design. Drug Discovery Today 7, 1056-1063.

13. Lipinski, C. A., Lombardo, F., Dominy, B. W., and Feeney, P. J. (1997) Experimental and computational approaches to estimate solubility and permeability in drug discovery and development settings. Adv. Drug Delivery Rev. 23, 3-25.

14. Feng, B., Mills Jessica, B., Davidson Ralph, E., Mireles Rouchelle, J., Janiszewski John, S., Troutman Matthew, D., and de Morais Sonia, M. (2008) In vitro P-glycoprotein assays to predict the in vivo interactions of P-glycoprotein with drugs in the central nervous system. Drug Metab. Dispos. 36, 268-275.

15. Whalen, K., Gobey, J., and Janiszewski, J. (2006) A centralized approach to tandem mass spectrometry method development for high-throughput ADME screening. Rapid Commun. Mass Spectrom. 20, 1497-1503.

16. Abad-Zapatero, C. (2007) Ligand efficiency indices for effective drug discovery. Expert Opin. Drug Discovery 2, 469-488.

17. Keserue, G. M., and Makara, G. M. (2009) The influence of lead discovery strategies on the properties of drug candidates. Nat. Rev. Drug Discovery 8, 203-212.

18. Hughes, J. D., Blagg, J., Price, D. A., Bailey, S., DeCrescenzo, G. A., Devraj, R. V., Ellsworth, E., Fobian, Y. M., Gibbs, M. E., Gilles, R. W., Greene, N., Huang, E., Krieger-Burke, T., Loesel, J., Wager, T., Whiteley, L., and Zhang, Y. (2008) Physiochemical drug properties associated with in vivo toxicological outcomes. Bioorg. Med. Chem. Lett. 18, 4872-4875.

19. Ertl, P., Rohde, B., and Selzer, P. (2000) Fast calculation of molecular polar surface area as a sum of fragment-based contributions and its applications to the prediction of drug transport properties. J. Med. Chem. 43, 3714-3717.

20. Waring, M. J. (2009) Defining optimum lipophilicity and molecular weight ranges for drug candidates-Molecular weight dependent lower log D limits based on permeability. Bioorg. Med. Chem. Lett. 19, 2844-2851.

21. Ploemen, J.-P. H. T. M., Kelder, J., Hafmans, T., van de Sandt, H., van Burgsteden, J. A., Salemink, P. J. M., and van Esch, E. (2004) Use of physicochemical calculation of $\mathrm{pKa}$ and CLogP to predict phospholipidosis-inducing potential: A case study with structurally related piperazines. Exp. Toxicol. Pathol. 55, 347-355.

22. Gao, H., Yao, L., Mathieu, H. W., Zhang, Y., Maurer, T. S., Troutman, M. D., Scott, D. O., Ruggeri, R. B., and Lin, J. (2008) In silico modeling of nonspecific binding to human liver microsomes. Drug Metab. Dispos. 36, 2130-2135.

23. Hosea, N. A., Collard, W. T., Cole, S., Maurer, T. S., Fang, R. X., Jones, H., Kakar, S. M., Nakai, Y., Smith, B. J., Webster, R., and Beaumont, K. (2009) Prediction of human pharmacokinetics from preclinical information: Comparative accuracy of quantitative prediction approaches. J. Clin. Pharmacol. 49, 513-533.

24. Van de Waterbeemd, H., Smith, D. A., and Jones, B. C. (2001) Lipophilicity in PK design: Methyl, ethyl, futile. J. Comput.-Aided Mol. Des.n 15, 273-286.
25. Kaye, C. M., Haddock, R. E., Langley, P. F., Mellows, G., Tasker, T. C. G., Zussman, B. D., and Greb, W. H. (1989) A review of the metabolism and pharmacokinetics of paroxetine in man. Acta Psychiatr. Scand., Suppl. 350, 60-75.

26. Leach, A. R., Hann, M. M., Burrows, J. N., and Griffen, E. J. (2006) Fragment screening: An introduction. Mol. BioSyst. 2, 429-446.

27. Houck, K. A., and Kavlock, R. J. (2008) Understanding mechanisms of toxicity: Insights from drug discovery research. Toxicol. Appl. Pharmacol. 227, 163-178.

28. Miller, V. P., Stresser, D. M., Blanchard, A. P., Turner, S., and Crespi, C. L. (2000) Fluorometric high-throughput screening for inhibitors of cytochrome P450. Ann. N. Y. Acad. Sci. 919, 26-32.

29. Fermini, B., and Fossa, A. A. (2003) The impact of druginduced QT interval prolongation on drug discovery and development. Nat. Rev. Drug Discovery 2, 439-447.

30. Deacon, M., Singleton, D., Szalkai, N., Pasieczny, R., Peacock, C., Price, D., Boyd, J., Boyd, H., Steidl-Nichols, J. V., and Williams, C. (2007) Early evaluation of compound QT prolongation effects: A predictive 384-well fluorescence polarization binding assay for measuring hERG blockade. J. Pharmacol. Toxicol. Methods 55, 238-247.

31. Leeson, P. D., and Springthorpe, B. (2007) The influence of drug-like concepts on decision-making in medicinal chemistry. Nat. Rev. Drug Discovery 6, 881-890.

32. Walum, E., Hedander, J., and Garberg, P. (2005) Research perspectives for pre-screening alternatives to animal experimentation. Toxicol. Appl. Pharmacol. 207, S393-S397.

33. JMP. (1989-2007) SAS JMP 7, SAS Institute, Inc. Cary, NC.

34. Spotfire. (1996-2007) Spotfire Decision Site 9, TIBCO Softrware Inc., Somerville, MA.

35. Hop, C. E. C. A., Cole, M. J., Davidson, R. E., Duignan, D. B., Federico, J., Janiszewski, J. S., Jenkins, K., Krueger, S., Lebowitz, R., Liston, T. E., Mitchell, W., Snyder, M., Steyn, S. J., Soglia, J. R., Taylor, C., Troutman, M. D., Umland, J., West, M., Whalen, K. M., Zelesky, V., and Zhao, S. X. (2008) High throughput ADME screening: Practical considerations, impact on the portfolio and enabler of in silico ADME models. Curr. Drug Metab. 9, 847-853. 